\title{
Biología reproductiva y placentotrofía en lagartijas del género Mabuya
}

\author{
Martha Patricia Ramírez-Pinilla \\ Laboratorio de Biología Reproductiva de Vertebrados, \\ Escuela de Biología, Universidad Industrial de Santander, Bucaramanga, Colombia
}

\begin{abstract}
Resumen
Presento una revisión de los trabajos que desde mi laboratorio se han venido realizando sobre la biología reproductiva y la placentotrofía en varias poblaciones de lagartijas vivíparas placentotróficas del género Mabuya. En este grupo la ovogénesis produce huevos microlecitos y en relación a ello se encuentra la mayor complejidad placentaria conocida hasta ahora entre Reptilia que converge en muchos aspectos morfológicos y fisiológicos con la que se conoce en mamíferos euterios; sin embargo, también muestra características exclusivas muy interesantes. Esta complejidad placentaria permite una masiva transferencia desde el oviducto materno de todos los nutrientes necesarios para el desarrollo de los embriones durante los 9 a 10 meses que dura su gestación. En esta revisión muestro la manera como una pregunta de investigación inicialmente sencilla ha permitido ahondar en la biología de este particular clado y cómo las nuevas herramientas que están ahora disponibles a las ciencias biológicas nos permitirán responder preguntas fundamentales en un futuro próximo.
\end{abstract}

Palabras clave: lecitotrofía, matrotrofía, oogenesis, placentación, reptiles escamados, viviparidad

Reproductive biology and placentotrophy in lizards of the genus Mabuya

\begin{abstract}
I present a review of the studies that have been made from my laboratory about the reproductive biology and placentotrophy of populations of the viviparous and placentotrophic lizards of Mabuya genus. In this group the oogenesis produces microlecithal eggs and related to that, it is found the highest degree of placental complexity yet known among Reptilia, which converge in several morphological and physiological aspects with those known in eutherian mammals; however, it also shows very interesting exclusive features. This placental complexity allows the transfer from the maternal oviduct of all the needed nutrients for embryo development during the 9 to 10 months of gestation. In this review I demonstrate the manner in which a research question initially simple has permitted get deeper in the biology of this particular clade and how the new tools that are now available to the biological sciences will enable us to answer fundamental questions in the near future.
\end{abstract}

Key words: Lecitotrophy, matrotrophy, oogenesis, placentation, squamate reptiles, viviparity

Dentro de las varias especies en las que se han hecho estudios desde mi laboratorio, en los últimos 15 años hemos estado trabajando junto con otros investigadores y estudiantes en la biología reproductiva de poblaciones de la lagartija vivípara del género Mabuya (Figura 1a). De las lagartijas presentes en Colombia, tan sólo dos clados distantes tienen la viviparidad como modo de paridad (dos especies del género Diploglossus, familia Anguidae y las poblaciones del género Mabuya, familia Scincidae), todas las demás lagartijas son especies ovíparas. Las lagartijas del género Mabuya estaban agrupadas para ese entonces (1998) como Mabuya mabouya, nominación que se aplicaba para todas las poblaciones del territorio continental colombiano y eran las únicas lagartijas vivíparas a las que teníamos relativo fácil acceso. Mi estudiante por ese entonces, hoy profesora del Departamento de Biología de la Universidad Nacional de Colombia, Dra. Adriana Jerez, se entusiasmó con el tema y decidimos que su trabajo de grado sería justamente analizar la morfología del desarrollo de las membranas extraembrionarias con base en ejemplares de las colecciones colombianas (Jerez, 1998). El trabajo resultó muy arduo pero gratificante, no es fácil trabajar con material no fijado para análisis histológico, así que muchos de los detalles morfológicos fundamentales se

\section{Correspondencia:}

Martha Patricia Ramírez-Pinilla, mpramir@uis.edu.com,mpramir@gmail.com Recibido: 6 de mayo de 2014

Aceptado: 3 de julio de 2014 
pierden. Con base en este trabajo y lo interesante que había resultado decidimos que el tema ameritaba continuarlo, obtuve financiación para realizar un proyecto de investigación colectando material de una población de este grupo (en la vereda Guadualito del municipio de Yacopí, Cundinamarca) que la Prof. Olga Castaño del ICN me había indicado era suficientemente abundante para obtener varios ejemplares para un estudio completo de su biología reproductiva. Desarrollamos el proyecto que nos permitió obtener machos y hembras de esta población durante un año de visitas periódicas a esta zona. El objetivo inicial era estudiar la dieta y uso de microhábitat y la actividad reproductiva anual de los machos y hembras de esta población. Estos fueron dos trabajos de grado de mis estudiantes, codirigidos por mi colega ecólogo Dr. Víctor Hugo Serrano, y que posteriormente fueron publicados (Ramírez-Pinilla et al., 2002; Caicedo-Portilla et al., 2010).

\section{Actividad reproductiva}

Sorprendentemente encontramos que esta población de lagartijas estaba conformada por dos grupos de hembras que se reproducían simultáneamente, las hembras primíparas que alcanzaban el tamaño para su primera reproducción a una edad aproximada de 3 meses (60-74 mm longitud rostro cloaca) y continuaban creciendo durante la gestación, y las hembras multíparas mayores que exhibían un crecimiento más limitado. La reproducción de las hembras parecía que era no estacional y asincrónica porque teniendo una gravidez prolongada (9-10 meses) en todos los meses se encontraban hembras preñadas conteniendo embriones en diferentes estados de desarrollo. Sin embargo, los nacimientos ocurrían solamente en los meses secos del año (enero-febrero y agostoseptiembre) lo que sugiere que tienen un patrón bimodal de reclutamiento de los neonatos a la población (Figura 1b). Las hembras multíparas tenían un patrón de crecimiento folicular preovulatorio y estaban listas para la cópula y un nuevo evento reproductivo inmediatamente después del parto, un patrón bimodal otra vez, mientras que los machos producían espermatozoides durante todo el año, estando disponibles a las dos subpoblaciones de hembras permanentemente (Figura 1b, Ramírez-Pinilla et al., 2002). Esta distribución temporal reproductiva en las hembras resultó ser sorprendente y muy particular. Otras poblaciones del género estudiadas en Brasil habían demostrado ser fuertemente estacionales en su reproducción, teniendo solo un pico anual de reclutamiento de neonatos (Rocha \& Vrcibradic, 1999; Vrcibradic \& Rocha, 1998).

Otro hallazgo interesante en este trabajo es que el tamaño de los ovocitos a la ovulación en la población de Mabuya estudiada es de $1.5 \mathrm{~mm}$ de diámetro (Figura 2a), similar a otras dos especies de Mabuya en donde se había hecho esta observación unos años antes (Vitt \& Blackburn, 1983, 1991; Blackburn \& Vitt, 1992). Los huevos de Reptilia se caracterizan por ser huevos con una masiva cantidad de yema o huevos telolecitos (del gr. telos, fin + lekithos, yema o vitelo); sin embargo en este clado, y como una particularidad importante asociada con la viviparidad, los huevos no acumulan casi yema y por tanto se les ha denominado como huevos microlecitos (con poca yema) y son, junto con los huevos de un clado africano placentotrófico obligado, los huevos más pequeños conocidos entre Reptilia (Tabla 1).
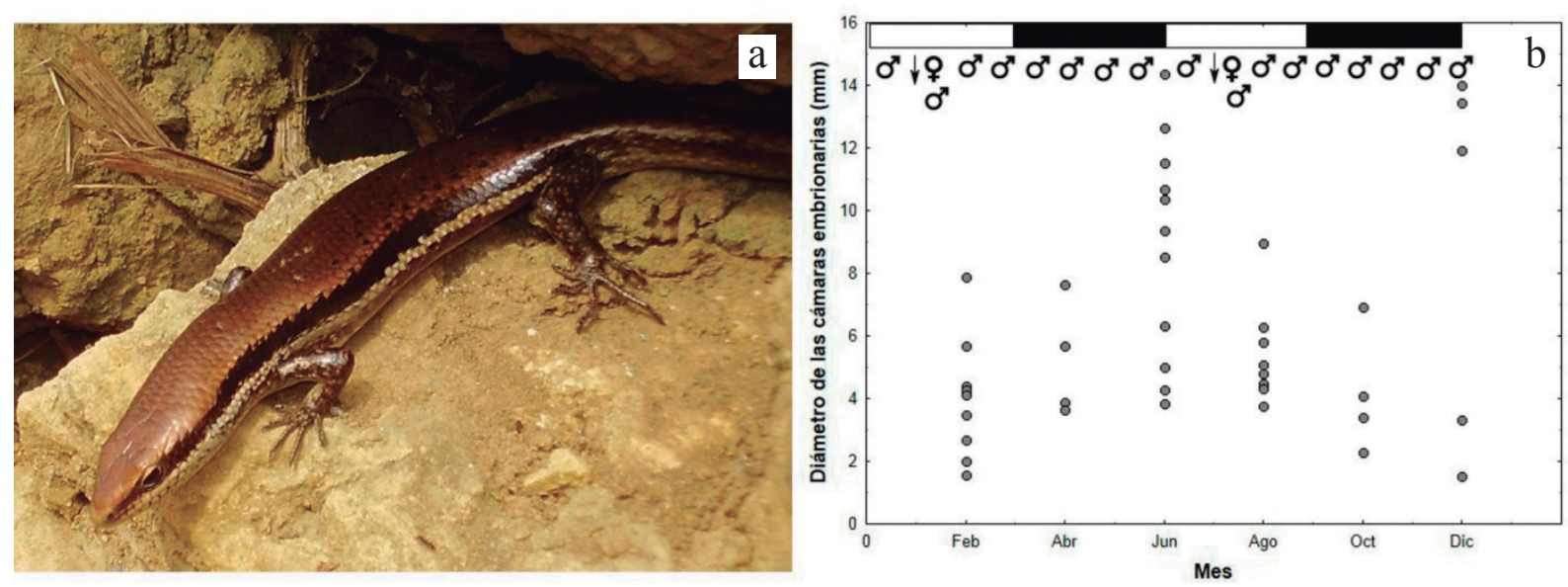

Figura 1. a. Hembra de la población de Mabuya de Curití, Santander. b. Resumen de la actividad reproductiva de la población de Yacopí, Cundinamarca. Se encuentran hembras gestantes durante todo el año; las cámaras embrionarias conteniendo los embriones más avanzados y de tamaño similar a los neonatos se encuentran en los meses de junio y diciembre al final de la temporada de lluvias (barras negras en la parte superior de la gráfica); los neonatos (flechas) aparecen durante las estaciones secas (barras blancas), así mismo las hembras son activas reproductivamente casi inmediatamente se dan los partos (signo femenino). Los machos se encuentran produciendo espermatozoides durante todo el año y por tanto son un recurso reproductivo permanente para las hembras (signo masculino). 

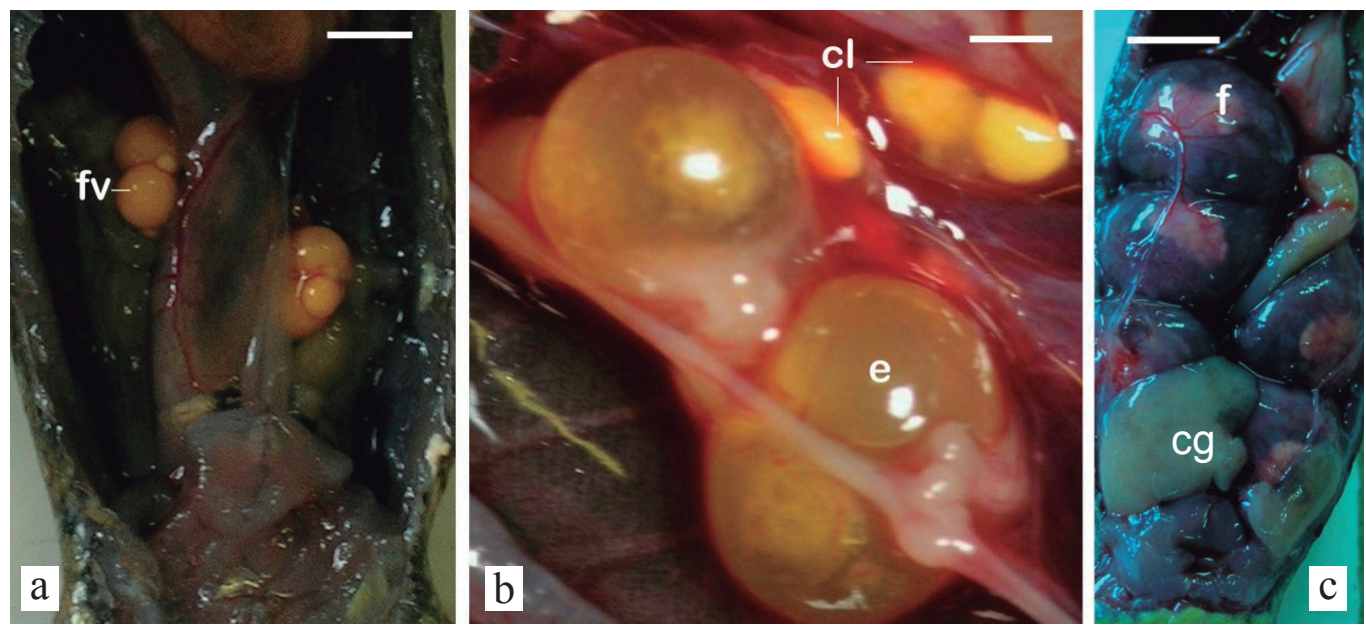

Figura 2. Observación macroscópíca de los tractos reproductivos de hembras de Mabuya en diferentes estados reproductivos. a. Hembra reproductiva con folículos vitelogénicos de tamaño cercano a la ovulación. fv, folículos vitelogénicos. Barra $=5 \mathrm{~mm}$. b. Hembra gestante con embriones oviductales en estado de gástrula. Se observa el contenido de la cámara embrionaria con un embrión mostrando una gota de grasa y el resto del contenido es acuoso producto de la hidratación una vez se da la ovulación. e, embrión; cl, cuerpos lúteos. Barra = $1 \mathrm{~mm}$. c. Hembra gestante con cámaras embrionarias conteniendo fetos ya pigmentados y próximos a nacer. f, fetos; cg, cuerpos grasos abdominales. Barra $=10 \mathrm{~mm}$.

Tabla 1. Tamaño de los huevos a la ovulación en diferentes amniotas. Se compara respecto del modo de paridad, patrón de nutrición embrionario y longitud corporal de la madre.

\begin{tabular}{|c|c|c|c|c|c|c|c|}
\hline & & Especie & $\begin{array}{l}\text { Modo de } \\
\text { paridad }\end{array}$ & Patrón de nutrición embrionaria & $\begin{array}{c}\text { Diámetro del } \\
\text { ovocito a la } \\
\text { ovulación (mm) }\end{array}$ & $\begin{array}{l}\text { Longitud } \\
\text { corporal de } \\
\text { la hembra }\end{array}$ & Fuente \\
\hline \multirow{10}{*}{ Reptilia } & \multirow{3}{*}{ Aves } & Mellisuga helenae & Oviparidad & Lecitotrofia, ovíparos altriciales & $6 \mathrm{~mm}$ & $60 \mathrm{~mm}$ & 1 \\
\hline & & Parus ater & Oviparidad & Lecitotrofía, ovíparos altriciales & $18 \mathrm{~mm}$ & $110 \mathrm{~mm}$ & 1 \\
\hline & & Struthio camelus & Oviparidad & Lecitotrofía, ovíparos precociales & $220 \mathrm{~mm}$ & $3000 \mathrm{~mm}$ & 1 \\
\hline & Crocodylia & Caiman crocodilus & Oviparidad & Lecitotrofía, ovíparos precociales & $70 \mathrm{~mm}$ & $770 \mathrm{~mm}$ & 2 \\
\hline & \multirow{6}{*}{ Squamata } & Gonatodes albogularis & Oviparidad & Lecitotrofía & $6 \mathrm{~mm}$ & $35 \mathrm{~mm}$ & 3 \\
\hline & & Niveoscincus coventryi & Viviparidad & Placentotrofía facultativa & $6 \mathrm{~mm}$ & $50 \mathrm{~mm}$ & 4 \\
\hline & & Pseudemoia entrecasteauxii & Viviparidad & Placentotrofía obligada & $6 \mathrm{~mm}$ & $55 \mathrm{~mm}$ & 5 \\
\hline & & Chalcides chalcides & Viviparidad & Placentotrofía obligada & $3 \mathrm{~mm}$ & $130 \mathrm{~mm}$ & 6 \\
\hline & & Mabuya spp & Viviparidad & Placentotrofía obligada & $1-2 \mathrm{~mm}$ & $80 \mathrm{~mm}$ & 7 \\
\hline & & Eumecia anchietae & Viviparidad & Placentotrofía obligada & $1 \mathrm{~mm}$ & $160 \mathrm{~mm}$ & 8 \\
\hline \multirow{3}{*}{ Mammalia } & Monotremata & Ornithorhynchus anatinus & Oviparidad & Lecitotrofía y matrotrofía postparto & $11 \mathrm{~mm}$ & $430 \mathrm{~mm}$ & 9 \\
\hline & Marsupialia & Monodelphis domestica & Viviparidad & Placentotrofía y matrotrofía postparto & $0,15 \mathrm{~mm}$ & $140 \mathrm{~mm}$ & 10 \\
\hline & Eutheria & Homo sapiens & Viviparidad & $\begin{array}{l}\text { Placentotrofía obligada y matrotrofía } \\
\text { postparto }\end{array}$ & $0,15 \mathrm{~mm}$ & $1650 \mathrm{~mm}$ & \\
\hline
\end{tabular}

(1), Dike y Kaiser, 2010; (2), Guerrero et al., 2003; (3), Serrano-Cardozo et al., 2007; (4), Murphy et al., 2006; (5), Thompson et al., 2002; (6), Blackburn y Callard, 1997; (7) Blackburn et al., 1983; Vitt y Blackburn, 1992; Hernández-Franyutti et al., 2005; Ramírez-Pinilla, 2006; (8), Flemming y Branch, 2003; (9), Grant, 2007; (10), Selwood, 2007.

En las lagartijas de zonas estacionales ya sea en la zona templada o en el trópico se encuentra una relación entre la utilización de los cuerpos grasos abdominales almacenados por las hembras en periodos no reproductivos y el proceso de la vitelogénesis. Este proceso permite el llenado de los folículos ováricos con lipoproteínas, vitelo o yema, que servirán para la nutrición embrionaria durante el desarrollo. Teniendo una producción de huevos microlecitos, se observó que al 
examinar la acumulación de cuerpos grasos abdominales en las hembras de nuestra población de Mabuya, la acumulación de las grasas se da durante la gestación y su depleción ocurre no durante el proceso de vitelogénico como en otras especies sino en la última fase de la gestación, cuando las hembras tienen su cavidad abdominal completamente distendida por la presencia de entre 3 y 7 embriones en fase de crecimiento fetal y cuando no consumen alimento (Figura 2c, CaicedoPortilla et al., 2010). Por lo tanto, en esta población la relación de reservas energéticas y la reproducción era diferente a la conocida para otras especies de lagartijas; la necesidad de energía y moléculas constructoras para la reproducción era fundamental durante la gestación y no durante el periodo preovulatorio en la vitelogénesis. Esto demostraba que para este clado vivíparo, el modo de nutrición embrionaria no se basaba en las reservas acumuladas en el vitelo (lecitotrofía) sino en la transferencia maternal de los nutrientes durante el desarrollo embrionario intrauterino (matrotrofía) a través de las membranas extraembrionarias y la formación de una placenta activa en la transferencia de estos nutrientes (placentotrofía).

\section{Placentación}

La morfología e histología de las membranas extraembrionarias que permiten la formación de una placenta activa y madura había sido estudiada con base en tejidos no propiamente fijados para análisis histológico para especies brasileñas de Mabuya (Blackburn, 1992) y por nosotros en el trabajo de grado mencionado previamente. Las lagartijas de la población de Yacopí colectadas para estos dos trabajos nos dieron la oportunidad de afinar el trabajo de grado de la por entonces Bióloga Adriana Jerez y comenzar una nueva línea de trabajo con otra estudiante de pregrado, utilizando esta vez tejidos especialmente fijados para trabajo histológico (Jerez \& Ramírez-Pinilla, 2001, 2003; Gómez \& Ramírez-Pinilla, 2004).

La morfología de placenta madura de las poblaciones estudiadas de Mabuya demostró ser mucho más compleja e interesante de lo que Blackburn en sus trabajos había sugerido. Antes de comentar la particularidad hallada por nosotros hay que hacer un recuento de conceptos básicos que permitirán entender los hallazgos mencionados. La evolución del huevo amniota, que fue un paso fundamental en la conquista del ambiente terrestre por parte de los vertebrados, involucra la evolución de cuatro membranas que se desarrollan por fuera del cuerpo del embrión pero derivan de él, las membranas extraembrionarias (Figura 3a). Estas se desarrollan tanto en amniotas ovíparos como en vivíparos y en amniotas lecitotróficos como placentotróficos. De ellas el corion interviene en el intercambio de gases y agua con el ambiente, el amnios establece un ambiente específico para el desarrollo embrionario, el alantoides permite el almacenamiento de

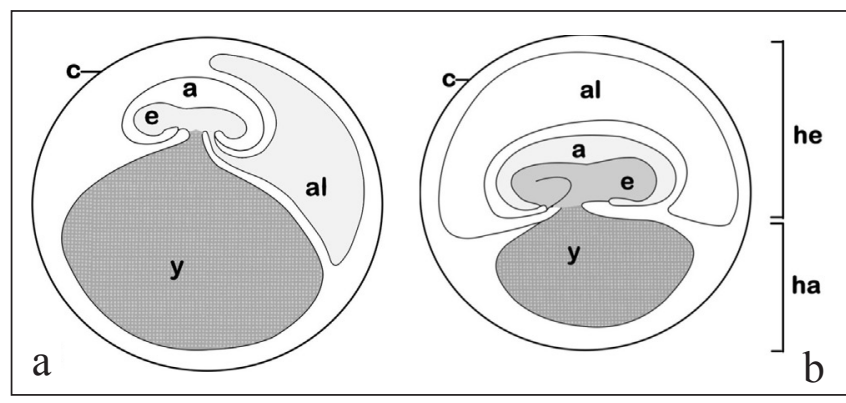

Fig. 3. a. Membranas extraembrionarias en amniotas. a, amnios; al, alantoides; c, corion; e, embrión; y, saco de la yema. b. En los escamados la posición relativa de las membranas extraembrionarias respecto de la posición del embrión define dos hemisferios dentro de la cámara embrionaria delimitada por el corion: En el hemisferio embrionario (he) se ubica el embrión (e) en desarrollo envuelto en el amnios (a); asimismo el alantoides (al) se expande por encima de la región dorsal del embrión adosándose al corion (c). En el hemisferio abembriónico (ha) se localiza el saco de la yema que en reptiles lecitotróficos y placentotróficos no extremos contiene el vitelo del que se nutrirá el embrión. En Mabuya este saco de la yema es pequeño, no contiene yema y por el contrario aparece lleno de material acuoso, como es observado también en mamíferos euterios.

desechos y, en los amniotas vivíparos lecitotróficos, el saco de la yema permite la transferencia de los nutrientes al embrión a partir de la yema (Thompson et al., 2002).

En los amniotas vivíparos placentotróficos (mamíferos euterios y cinco clados de lagartijas de la familia Scincidae, uno de ellos Mabuya) la provisión de los nutrientes que es posterior a la ovulación ocurre a partir del oviducto materno hacia el embrión atravesando por al menos una de estas membranas extraembrionarias. La conjunción de los tejidos maternos, el oviducto, y las membranas extraembrionarias forman lo que denominamos placenta. La placenta permite entonces la interacción de órganos fetales (las membranas extraembrionarias) y maternales (el oviducto) para permitir el intercambio fisiológico.

La organización de las membranas extraembrionarias respecto del oviducto materno permite definir dos tipos de placentas en amniotas: 1) onfaloplacentas que son las derivadas de la conjunción del saco de la yema y el corion y se ubican en el hemisferio abembriónico, y 2) las alantoplacentas formadas por la conjunción del alantoides con el corion, y su relación con el oviducto materno y se ubican en el hemisferio embriónico (Figura 3b). Las onfaloplacentas son características de las placentas en la mayoría de los lagartos vivíparos lecitotróficos y placentotróficos, en los mamíferos marsupiales en donde permanecen durante la mayor parte de su breve desarrollo embrionario, y son sólo transitorias en los lagartos vivíparos placentotróficos extremos como en Mabuya. Las alantoplacentas son características de los 
mamíferos euterios en donde son las que permiten el masivo intercambio de nutrientes propio de este grupo y también se encuentran en todos los lagartos vivíparos principalmente implicadas en el intercambio gaseoso.

En Mabuya, la alantoplacenta o placenta derivada del corioalantoides es morfológica y fisiológicamente la más especializada placenta conocida entre Reptilia y converge en el grado de complejidad y de placentotrofía con la conocida en los mamíferos euterios. La placenta madura de Mabuya tiene especializaciones morfológicas complejas exclusivas de este clado: 1) un placentoma altamente elaborado con una fuerte interdigitación de los tejidos maternos y fetales, en donde se destaca un arreglo sincitial del epitelio uterino frente a un epitelio coriónico biestratificado especializado; 2) una zona que rodea al placentoma, denominada paraplacentoma, delimitada por un pliegue coriónico; 3) areolas coriónicas que reciben materiales nutritivos del epitelio uterino a partir especialmente de glándulas endometriales, 4) placas de absorción o áreas redondeadas $\mathrm{y}$ pequeñas formadas por células coriónicas hipertrofiadas en aposición a células epiteliales columnares secretoras del endometrio; 5) segmentos respiratorios, o áreas de atenuación de los epitelios del corion y endometriales de manera que los capilares sanguíneos uterinos y del corioalantoides quedan en una muy cercana proximidad (Figura 4 a, b, c). Las cuatro primeras especializaciones de la alantoplacenta estarían relacionadas con el intercambio de materiales nutritivos por procesos como la histotrofía, la quinta especialización con el intercambio de gases entre el embrión y el útero materno (Jerez \& Ramírez-Pinilla, 2001; Ramírez-Pinilla, 2010). Cabe resaltar que ninguna placenta de mamíferos euterios reúne simultáneamente toda esta serie de especializaciones morfológicas como las que se encuentran en Mabuya.

Ya que contábamos con hembras preñadas en todos los estados de gestación, decidimos estudiar cómo era el desarrollo de esta placenta compleja y si el desarrollo de la placentación en este clado difería del resto de los reptiles y de mamíferos (Jerez \& Ramírez-Pinilla, 2003). Los análisis topológicos e histológicos de las cámaras de incubación (los embriones en sus membranas extraembrionarias dentro del oviducto materno) mostraron una secuencia de desarrollo de membranas embrionarias que es compartida por todos los amniotas y una secuencia placental similar con otros reptiles escamados. Eso implica que el patrón de desarrollo de las membranas extraembrionarias sería evolutivamente conservativo; básicamente las placentas de los amniotas son similares debido a que se constituyen por tejidos homólogos. Sin embargo, éstas han evolucionado independientemente en múltiples oportunidades siguiendo trayectorias convergentes o paralelas (Blackburn, 1992; Stewart y Thompson, 2003). Se encontró que en Mabuya la secuencia placental (placenta bilaminar del saco de la yema, corioplacenta y alantoplacenta)
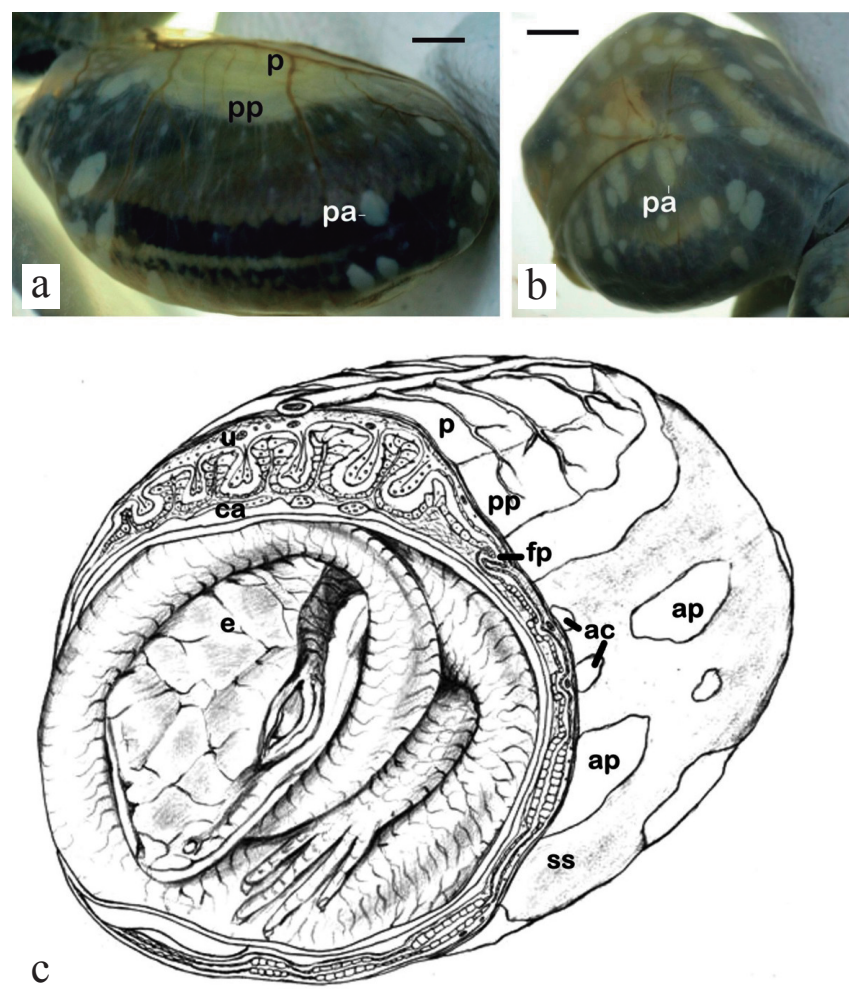

Figura 4. Cámara embrionaria de Mabuya en estados avanzados de gestación durante la fase de crecimiento fetal. a. La fotografía muestra el hemisferio embriónico de la cámara donde se pueden observar el placentoma (p), paraplacentoma (pp) y las placas de absorción (pa) de diferentes tamaños inmersas en los segmentos respiratorios. Se ve claramente la vascularización oviductal que irradia sobre el placentoma. Barra $=5 \mathrm{~mm}$. b. Se observa el hemisferio abembriónico de la cámara y el feto en vista ventral. No se distingue saco de la yema pues es muy pequeño y está unido al cuerpo del embrión. Se pueden distinguir claramente las placas de absorción derivadas del corioalantoides que rodean por completo la cámara embrionaria. c. Interpretación gráfica de la cámara embrionaria mostrando las especializaciones de la alantoplacenta. ac, areolas coriónicas, ss, segmentos respiratorios; fp, pliegue del paraplacentoma que lo separa del resto de la cámara embrionaria.

permite la absorción de nutrientes desde el principio del desarrollo embrionario. Esto es, la placentotrofía ocurre desde el inicio mismo del desarrollo como así ocurre en mamíferos euterios, lo que indica que la placentotrofía en este clado es extrema y obligada: todo el desarrollo embrionario depende básicamente de la transferencia placental de nutrientes.

\section{Ovogénesis y placentación}

Este patrón de desarrollo embrionario es entonces concomitante con la reducción en la acumulación de vitelo en los ovocitos y la formación de huevos microlecitos. Por lo tanto, la ovogénesis en este clado debería tener cambios fundamentales. La pregunta entonces era, ¿cómo es la 
morfología ovárica y el proceso de ovogénesis en Mabuya? Nuestro trabajo entonces se enfocó en estudiar la ovogénesis inicialmente en las lagartijas de nuestra población de Yacopí (Gómez \& Ramírez-Pinilla, 2004). Dentro de los vertebrados hay varios clados en donde ha evolucionado la viviparidad y la nutrición embrionaria que reemplaza la lecitotrofía; por lo tanto, hay una supresión gradual de la síntesis de lipoproteínas de la yema en relación con la adquisición de recursos alternativos de aporte nutricional al embrión en desarrollo. Se ha observado que el grado de inhibición del proceso de la vitelogénesis se correlaciona directamente con el grado de complejidad de los mecanismos de transferencia de nutrientes desde el útero al embrión (Callard y Ho, 1987). El extremo de esta tendencia es la supresión total de la provisión de yema que se observa en los mamíferos euterios. Nuestro trabajo demostró que la estructura ovárica y el proceso de ovogénesis son conservados en Mabuya respecto de los vertebrados y particularmente respecto de los escamados. Los primeros estados del proceso de vitelogénico se conservaban; sin embargo, la vitelogénesis era corta sugiriendo que el proceso se truncaba, provocando la ovulación de huevos muy pequeños. Un hallazgo importante se relacionó con la pregunta de si los cuerpos lúteos, responsables de la secreción de progesterona y con ello del mantenimiento de los embriones dentro del oviducto durante la gestación, se mantenían durante toda la gestación como un recurso permanente de esta hormona o no. Los resultados mostraron que por el contrario, los cuerpos lúteos entraban en luteólisis (degradación) muy temprano durante el desarrollo de la gestación y que por tanto el recurso de progesterona debería ser aportado por algún tejido extraluteal, posiblemente la placenta como ocurre en mamíferos.

\section{Inferencia morfología-función}

Estos trabajos nos habían mostrado muy interesantes resultados pero deberíamos tener una mucha mejor resolución a nivel histológico que nos permitiera hacer más inferencias de morfología-función tanto para la placenta como para el ovario en este clado. Los tejidos de las hembras de la localidad de Yacopí también fueron fijados para trabajar con microscopía electrónica de transmisión. En este campo la experta era la profesora Gloria de Pérez. Por lo tanto, junto a ella y un par de estudiantes de pregrado realizamos estudios paralelos de la placenta madura y del ovario pero a nivel de microscopía óptica de alta resolución y de ultraestructura en los laboratorios de la Universidad Nacional de Colombia en Bogotá. Este nivel de resolución nos permitió afinar sustancialmente nuestras observaciones.

Para el caso de la placenta encontramos que cada una de las especializaciones descritas tendría funciones de absorción de nutrientes cada una con mecanismos particulares y posiblemente en la síntesis de hormonas y proteínas. Así, en el placentoma (Figura 5a) las características citológicas indican una transferencia de nutrientes a través de una extensiva red de microvellosidades interdigitadas entre los epitelios embrionario y uterino, en el paraplacentoma (Figura 5b) la transferencia de nutrientes pareciera centrarse en una masiva transferencia de lípidos y de derivados de glándulas endometriales; en las areolas coriónicas (Figura 5c) el epitelio coriónico absorbe grandes cantidades de secreciones tanto de las glándulas endometriales como de desechos celulares por lisis celular de algunas de las células de los epitelios uterino y coriónico; en las placas de absorción se establece un sistema de relación diferente entre los epitelios materno y fetal (Figura 5c), acá los epitelios están firmemente adheridos y sus ápices celulares exhiben gránulos electrodensos que pueden estar relacionados con funciones autocrinas o paracrinas; finalmente, en los segmentos respiratorios la poca distancia interhemal entre los tejidos maternos y fetales confirmaba su función en el intercambio respiratorio de gases. Así, las características citológicas e histológicas nos estaban mostrando que cada una de las especializaciones de la alantoplacenta de Mabuya tenía una relación diferente entre los tejidos maternos y fetales sugiriendo diferentes mecanismos localizados de transferencia y producción de sustancias entre madre y feto y posiblemente también en la otra dirección. Sin embargo, una característica común encontrada en toda la superficie de la cámara de incubación fue la presencia de gotas de lípidos en los epitelios interactuantes; esto sugiere que los lípidos pueden ser transferidos a través de toda la alantoplacenta independientemente de la especialización morfológica encontrada, y que la transferencia placental de lípidos puede ser la principal fuente de energía y recursos lipoprotéicos en este clado (Ramírez-Pinilla et al., 2006).

Con base en una característica morfológica interesante hallada en el placentoma, enfocamos nuestra atención a la ultraestructura de esta región que parecía la más compleja entre las especializaciones de la placenta de Mabuya. Para entonces habíamos encontrado una nueva población de Mabuya que nos proveería de más material para poder hacer comparaciones interpoblacionales. Esta población era de la zona del municipio de Curití en Santander, sustancialmente más cerca que nuestra antigua población de Mabuya. En el placentoma de cámaras embrionarias con embriones avanzados algunas células coriónicas que interdigitan mediante vellosidades y microvellosidades con el sincitio epitelial del útero invaden este sincitio mediante prolongaciones citoplasmáticas complejas. Estas prolongaciones entran en contacto con los capilares uterinos mediante un sistema complejo de doble membrana que rodea el capilar. Esta relación cercana entre la circulación materna y el corion sugiere que en el placentoma se forma una placenta endoteliocorial muy particular (Figura 6a, b). Este descubrimiento constituye el primer ejemplo documentado 

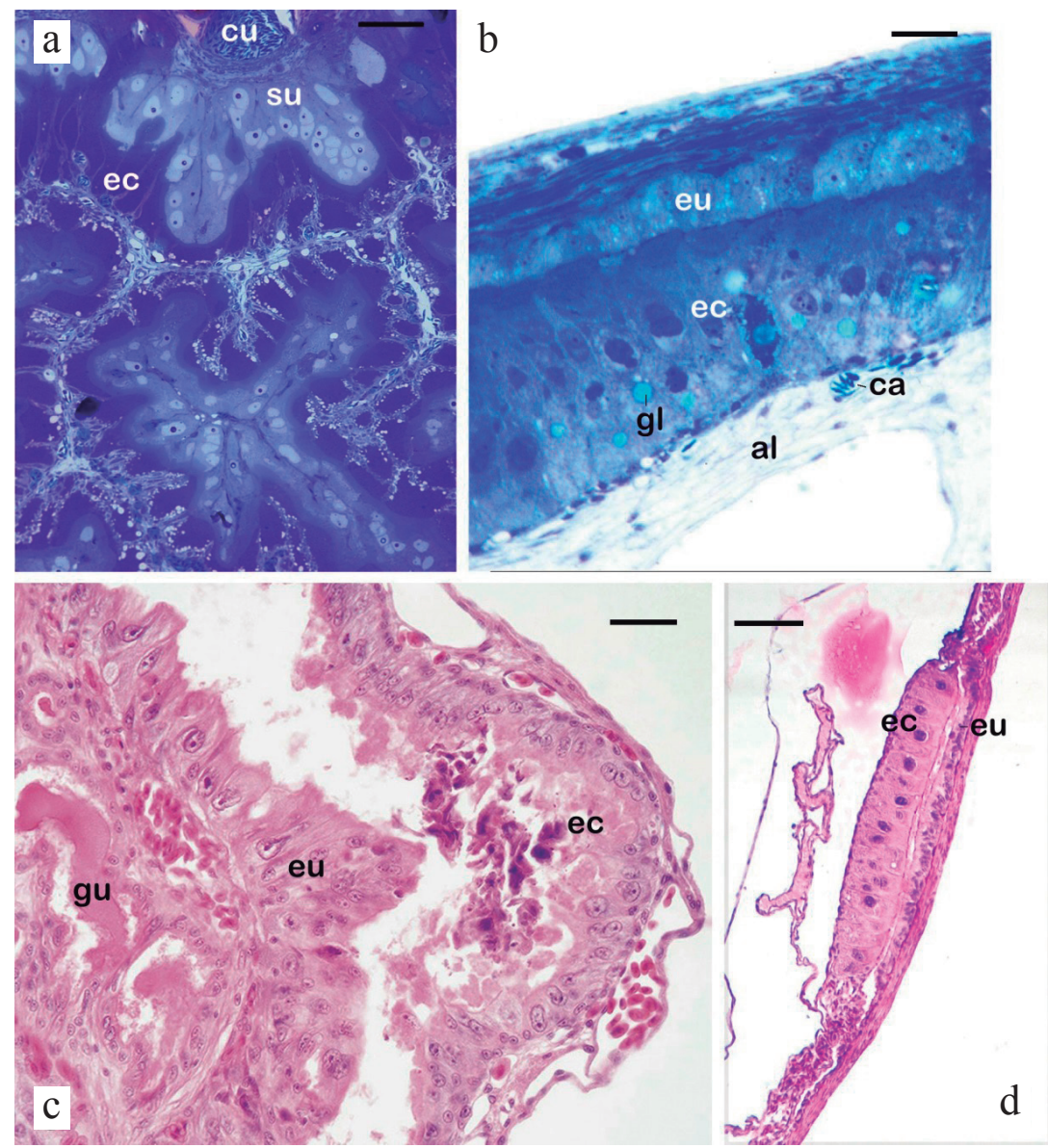

Figura 5. Cortes histológicos de las especializaciones de la alantoplacenta en Mabuya. a. Placentoma, se observa cómo interdigitan los tejidos embrionarios y maternos. cu, circulación uterina; su, sincitio epitelial uterino; ec, epitelio coriónico. Barra $=50 \mu \mathrm{m}$. b. En el paraplacentoma no se interdigitan los tejidos maternos y fetales, el paraplacentoma rodea al placentoma delimitándolo. Se observan varias gotas de lípidos (gl) en las altamente hipertróficas células del epitelio coriónico (ec) que se adosan al epitelio uterino (eu). Se observa tejido conjuntivo laxo del alantoides (al) y capilares alantoicos (ca) bajo el epitelio coriónico. Barra $=20 \mu \mathrm{m}$. c. En las areolas coriónicas se observan glándulas uterinas (gu) que liberan abundante material a la concavidad formada por el epitelio coriónico (ec). Las células del epitelio uterino (eu) se observan hipertrofiadas y pueden liberar parte de su citoplasma hacia el epitelio coriónico. Barra $=45 \mu \mathrm{m}$. d. Las placas de absorción son como botones de adherencia entre el epitelio uterino (eu) y el epitelio coriónico (ec) ambos epitelios son columnares e hipertrofiados, lo que contrasta con la zona de los segmentos respiratorios donde ambos epitelios son planos. Barra $=30 \mu \mathrm{m}$.

de este tipo de placentación entre Reptilia y que tampoco se ha descrito en otro clado con placentación compleja o con placentotrofía (Vieira et al., 2007).

Esta importante característica era compartida por las diferentes poblaciones de Mabuya estudiadas. Sin embargo, para corroborarlo buscamos tejidos placentarios de nuevas poblaciones de Mabuya en el país y fuera del país para determinar la variación interpoblacional e interespecífica placentaria. Acá el trabajo era comparativo y podríamos hacerlo nuevamente con material de museos y con histotecnia corriente. Este trabajo lo hicimos con otra estudiante de pregrado y fue publicado (Leal \& Ramírez-Pinilla, 2008).
Se encontró que para 12 poblaciones/especies del norte de Suramérica se conserva el patrón general de la placenta con las mismas especializaciones de la alantoplacenta; sin embargo, se registra una variación morfológica interesante. En algunas poblaciones las placas de absorción se encuentran altamente plegadas y delimitadas por una especie de anillo de cierre simulando pequeños placentomas, además se encontraron regiones altamente plegadas en los segmentos respiratorios. Las dos modificaciones halladas incrementan la superficie de relación entre los epitelios uterino y coriónico, sugiriendo cambios interesantes que pueden implicar variaciones en la eficiencia de cada una de las especializaciones de la alantoplacenta. 


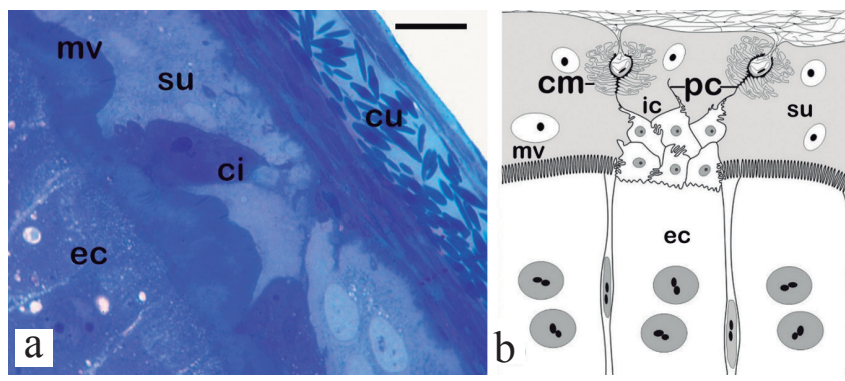

Figura 6. Células coriónicas invasoras del sincitio epitelial uterino del placentoma en Mabuya. a. Fotografía de un corte semifino de la región del placentoma que muestra el epitelio coriónico (ec) del que se desprenden las células invasoras (ci) que migran entre el sincitio uterino ( $\mathrm{su}$ ) y emiten proyecciones citoplasmáticas para alcanzar los capilares subepiteliales uterinos que derivan de la circulación uterina (cu) en el hemisferio embriónico. Barra $=25 \mu \mathrm{m}$. b. Interpretación gráfica derivada del estudio por microscopía electrónica de transmisión mostrando cómo las células invasoras (ci) emiten proyecciones citoplasmáticas $(\mathrm{pc})$ profundamente en el sincitio epitelial uterino y de ellas se desprende una compleja red de doble membrana $(\mathrm{cm})$ que envuelve el endotelio de los vasos sanguíneos uterinos que igualmente están inmersos dentro del sincitio.

El análisis de microscopía óptica de alta resolución y ultraestructural del ovario de las hembras de esta población (Vieira et al., 2010) permitió evidenciar que el ooplasma de los folículos en vitelogénesis muestra abundantes organelos membranosos con un centro de lipoproteínas, sin embargo no se encontraron plaquetas vitelinas organizadas como las características de los reptiles lecitotróficos. Por el contrario, hacia el ooplasma medular se encontraron grandes vesículas electrolúcidas. Esta morfología y contenido ooplasmático es muy similar al encontrado en los folículos ováricos preovulatorios de mamíferos marsupiales y euterios, lo que sugiere una muy interesante convergencia evolutiva relacionada con la evolución de la placentotrofía. En mamíferos estas vesículas han sido consideradas como elementos residuales cuya función es proveer de matriz extracelular, especialmente proteínas estabilizantes y ácido hialurónico que permiten la absorción de agua para la expansión del blastocisto en la primera fase del desarrollo embrionario, y por tanto no están relacionados con la nutrición embrionaria. En Mabuya, no conocemos el contenido de estas vesículas y por tanto se requiere su caracterización bioquímica. Sin embargo, inmediatamente después de ocurrida la ovulación hay un rápido incremento en volumen del huevo debido a la absorción de agua (Figura $3 b)$, lo que permite que la cámara embrionaria se forme y se desarrolle la primera membrana extraembrionaria que rodea el embrión en formación (formando la primera placenta en la ontogenia, la placenta bilaminar del saco de la yema). Así, se sugiere una función similar para estas vesículas a la demostrada para los mamíferos.

\section{Cuantificando la transferencia placental de nutrientes entre madre y embriones}

Conociendo la morfología se puede inferir la función, la morfología nos había indicado que había una gran complejidad cito-histológica que convergía con la de mamíferos euterios, los que igualmente son organismos placentotróficos obligados. Una placentotrofía obligada implicaba que el desarrollo embrionario en este clado se basaba en la transferencia placental de casi todas las moléculas constructoras del cuerpo del embrión y la energía necesaria para ello. Una cuantificación gruesa permitía corroborar que había una absorción neta de nutrientes (agua, iones: calcio, potasio, sodio, magnesio; nitrógeno y peso seco, Figura 7 a,b, y lípidos) durante la gestación y que la drástica reducción en los nutrientes de origen ovárico y así del tamaño del huevo estaba relacionada con una placentotrofía obligada desde los estados de desarrollo más temprano; por consiguiente, Mabuya se constituía en el clado con el máximo nivel de placentotrofía conocido entre Reptilia (Figura 7 c, d, Ramírez-Pinilla, 2006). La morfología sugería mecanismos y ya sabíamos qué nutrientes, cuándo y en qué cantidad estaban siendo transferidos entre la madre y los embriones en desarrollo. Sin embargo, necesitábamos hacer una cuantificación más fina detallando por ejemplo qué lípidos estaban siendo transferidos, si estos lípidos eran similares o no a los del vitelo de una especie lecitotrófica, o si el ovocito contenía un set de nutrientes diferentes a los que luego eran transferidos a través de la placenta. Para responder a estas nuevas preguntas fisiológicas necesitaba de colegas especializados y herramientas sofisticadas de cuantificación. Por lo tanto, aunamos esfuerzos con un estudiante de maestría, Elkin Rueda, y el laboratorio de la Profesora Dra. Elena Stashenko. Se miraron nuevamente el nitrógeno (como índice de proteínas) y los iones transferidos, pero también la cantidad de lípidos totales y sus fracciones (colesterol, vitamina $\mathrm{E}$ y ácidos grasos en sus mayores clases - triacilgliceroles, fosfolípidos, colesteril ésteres, ácidos grasos libres-) durante los diferentes estados de la gestación y en los recién nacidos (Ramírez-Pinilla et al., 2011).

Encontramos que los huevos recientemente ovulados en Mabuya contienen proporcionalmente más agua que en cualquier otro huevo reptiliano, lo que está relacionado con la pérdida de moléculas lecitotróficas en el huevo. Este contenido del oocito es aún más diluido cuando el huevo se hidrata en el oviducto al inicio del desarrollo embrionario. El agua es transferida entonces desde los primeros estados del desarrollo hasta el final de la gestación cuando esta transferencia es máxima. Asimismo, tanto las cantidades relativas y absolutas de iones inorgánicos son reducidos en el huevo de Mabuya respecto de otros reptiles, por lo tanto estos iones deben pasar a través de la placenta durante la gestación. 

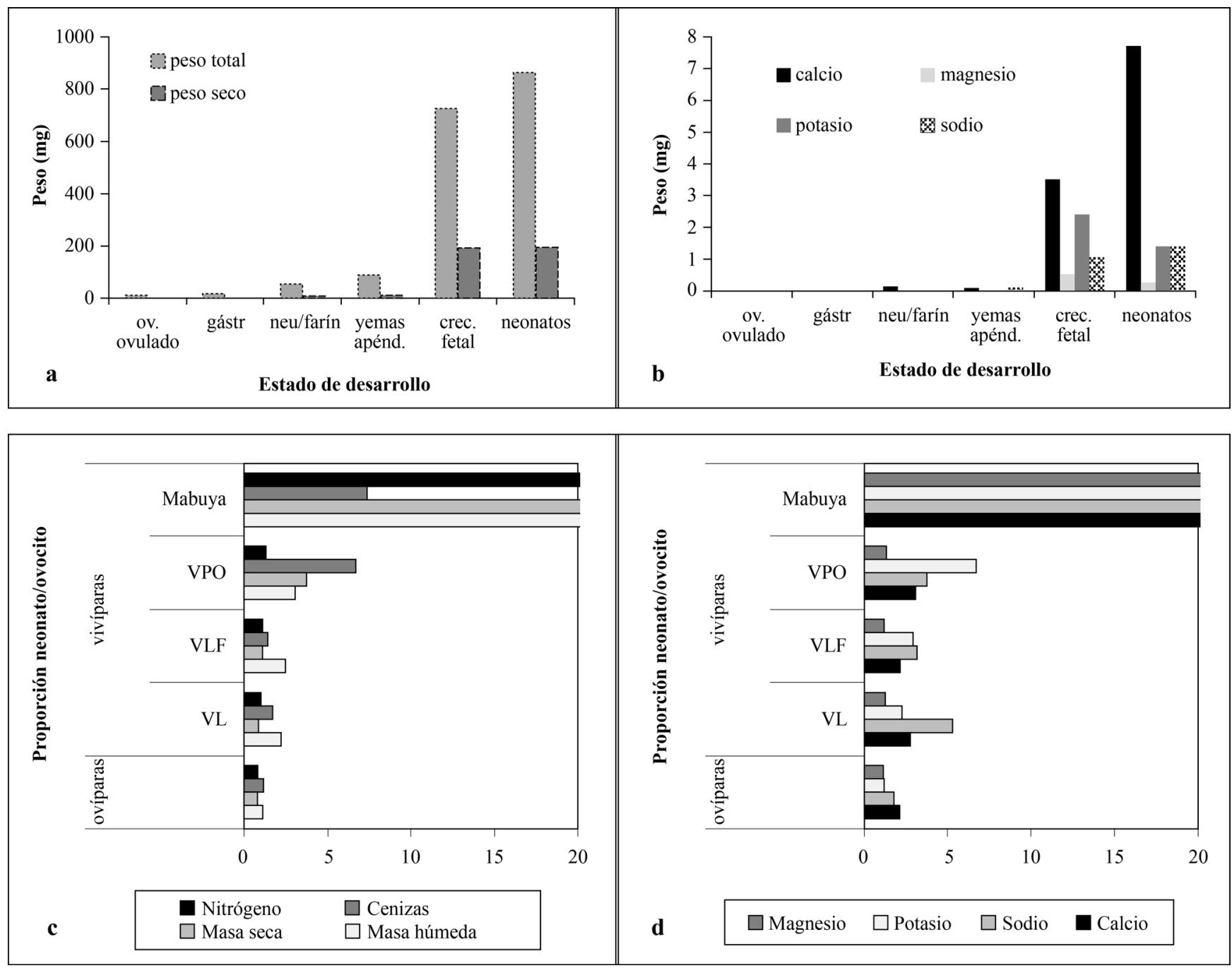

Figura 7. Placentotrofía en Mabuya. a. Variación en el peso total y peso seco durante los estados de desarrollo en la gestación y en el neonato. b. Variación en el contenido de iones calcio, magnesio, potasio y sodio durante los estados de desarrollo en la gestación y en el neonato. Ov. ovulado: ovocito recientemente ovulado; gást.: gástrula; neu/farín.: néurula a faríngula; yemas apend.: estado de desarrollo de las yemas de los apéndices de los miembros; crec. fetal: último estado de desarrollo en donde la organogénesis ha concluido y se da el crecimiento fetal. c. Comparación en la proporción neonato/ovocito (índice de placentotrofía) recién ovulado en diferentes grupos de reptiles escamados, incluyendo lagartijas ovíparas y vivíparas con viviparidad lecitotrófica (VL, la mayoría de las lagartijas vivíparas), viviparidad placentotrófica facultativa (VLF, lagartijas del género Niveoscincus de Australia), viviparidad placentotrófica obligatoria (VPO, Chalcides chalcides en el Mediterráneo y lagartijas australianas del género Pseudemoia) y en Mabuya. Los datos son tomados de Thompson et al. (2002). Aunque no hay datos aún el clado surafricano que incluye a Eumecia anchietae y Trachylepis ivensi podrían tener un mayor índice de placentotrofía que Mabuya por cuanto el tamaño corporal de los neonatos al nacimiento es mayor que el tamaño promedio en las especies de Mabuya.

Igualmente se encontró que los lípidos no son sólo reducidos en cantidad en el huevo microlecito de Mabuya cuando se compara con el contenido de los huevos en clados con menor grado de placentotrofía o con clados lecitotróficos, sino también en el contenido relativo de las diferentes clases de lípidos: los ovocitos de Mabuya tienen proporciones reducidas de lípidos energéticamente ricos (triacilgliceroles) y proporciones incrementadas de lípidos menos energéticamente ricos (fosfolífidos y colesteril ésteres). En ausencia de cáscara del huevo y teniendo un ooplasma pobre en iones, las lagartijas Mabuya deben transferir casi la totalidad del contenido iónico para el desarrollo a través de la placenta; esta transferencia es lenta durante el desarrollo temprano y la organogénesis y máxima al final de la gestación cuando se da el periodo de crecimiento fetal (Figura 7b). De manera similar, hay una transferencia masiva de lípidos en la fase de 
crecimiento fetal; se encontró que hay una absorción neta de todas las clases de lípidos analizadas pero sus proporciones relativas varían con el avance de la gestación. Esta variación refleja funciones particulares de cada una de esas clases de lípidos durante el desarrollo embrionario.

\section{Transportadores de nutrientes}

A este momento ya teníamos una idea muy clara de que la placentotrofía en Mabuya se daba para básicamente todas las moléculas y que a diferencia de mamíferos en donde la fuente energética primaria durante el desarrollo embrionario son los carbohidratos, en Mabuya esta fuente son los lípidos, los cuales son transferidos desde el inicio mismo del desarrollo. Por lo tanto, a pesar de una aparente convergencia morfológica y fisiológica, los mecanismos asociados a la placentotrofía entre estos dos clados son diferentes, demostrando una clara evolución independiente. La pregunta ahora era definir los mecanismos de transporte y los transportadores empleados para esta transferencia placental de nutrientes, cómo y cuándo durante el desarrollo estos nutrientes eran transferidos. La pregunta era aún más compleja y unimos esfuerzos con un placentólogo de mamíferos de la Universidad de Cambridge (Inglaterra) que tenía experiencia en imunocitoquímica, técnica que nos permitiría ver, utilizando moléculas conocidas como transportadores en mamíferos, si estas se expresaban en los tejidos placentales de Mabuya (Wooding et al., 2011). Pudimos localizar sistemas de transferencia de calcio (calbindinas $28 \mathrm{kD}$ y $9 \mathrm{kD}$ ), glucosa (transportadores de glucosa 1 y 8 ) y agua (acuaporinas 1 y 3 ). Los anticuerpos localizados se encontraron en varias de las especializaciones de la placenta madura, indicando que el transporte de esas moléculas se da por varias vías, sin embargo ninguno de estos transportadores fue localizado en los segmentos respiratorios, lo que prueba su función exclusiva en el intercambio gaseoso.

\section{El presente y futuro}

Los mecanismos moleculares de transporte de nutrientes son relativamente bien conocidos en mamíferos euterios y solo han sido investigados en lagartijas placentotróficas no extremas (Pseudemoia entrecasteauxii, P. spenceri, y Chalcides ocellatus, Biazik et al., 2009; Brandley et al., 2012; Griffith et al., 2013a, b). ¿Son los mecanismos de transporte de nutrientes similares entre clados que evolucionaron la placentotrofía de manera convergente? ¿La evolución de la placentotrofía y la placentación compleja derivan de los mismos juegos de genes en los clados de scincidos placentotróficos y en mamíferos? Aun cuando estábamos afinando nuestro conocimiento de los mecanismos de transporte, la inmunocitoquímica no era la técnica más eficaz para entender como era que funcionaban las placentas durante la gestación en Mabuya. Afortunadamente las herramientas biológicas han revolucionado nuestra posibilidad de entender y responder tantas preguntas complejas. Estas poderosas herramientas permiten dilucidar el funcionamiento de los órganos, tejidos y células en un momento dado. Básicamente se puede determinar qué porción del genoma es transcrito a ARN mensajero, es decir, qué material genético es expresado en un estado dado, son los análisis de transcriptómica. Recientemente iniciamos un trabajo en colaboración con mis estudiantes que ahora hacen su doctorado en la Universidad de Gainesville (USA), Francisca Leal y Óscar Tarazona, y la colaboración de su profesor Marty Cohn, en éste se está identificando la expresión génica completa de los transcriptomas de las placentas de Mabuya durante los diferentes estados de gestación usando análisis RNA-Seq. Los resultados preliminares están mostrando que hay unos muy claros paralelos en los procesos de expresión génica entre Mabuya y mamíferos euterios. Con nuevos genomas de reptiles escamados secuenciados se podrá investigar sobre la convergencia en las rutas hacia la viviparidad y placentotrofía. De la misma manera con mayor información genómica se podrá tener un mayor número de genes identificados en los trasncriptomas secuenciados, lo que permitirá entender y conocer los elementos que regulan la expresión de genes asociados a la placentotrofía (Van Dyke, 2014).

De forma paralela a todos estos trabajos, seguíamos con el problema de la identidad específica de las poblaciones que estábamos trabajando. Tampoco sabemos cómo estas poblaciones se relacionan entre sí, ni su historia biogeográfica. Con una estudiante de doctorado de la Universidad de los Andes pero egresada nuestra decidimos afrontar el problema. Nuevamente, las herramientas moleculares modernas nos permiten responder preguntas que otrora sólo la morfología tradicional lo permitían. Este clado es particularmente complejo porque su morfología externa es muy conservativa, por lo tanto las herramientas moleculares eran fundamentales para definir clados subgenéricos en Colombia. Nelsy Pinto, junto con otros colegas, ha desarrollado una filogenia con base en caracteres moleculares de genes mitocondriales y nucleares para las poblaciones colombianas de Mabuya. Este trabajo está por ser publicado. Igualmente en el futuro cercano tendremos la descripción de estos clados como nuevas especies de Mabuya con distribuciones definidas; estamos resolviendo otro viejo problema.

Después de más de 15 años de iniciar un trabajo con un grupo de lagartijas que pasaban desapercibidas para muchos, hemos podido entender una muy buena proporción de la biología reproductiva de este grupo. La complejidad placentaria y el grado de placentotrofía alcanzado hacen de Mabuya un modelo biológico de estudio precioso del que aún hay mucho por conocer.

\section{Agradecimientos}

Mis mayores agradecimientos a las siguientes instituciones y personas: La Universidad Industrial de Santander y la Universidad Nacional de Colombia financiaron parcialmente 
los estudios relacionados; igualmente, la Fundación para la Promoción de la Investigación y la Tecnología del Banco de la República y el Instituto Colombiano para el Desarrollo de la Ciencia y la Tecnología Francisco José de Caldas (COLCIENCIAS contratos 1102-05-13556 y1102-452-21152).

Por el préstamo de ejemplares de colección a V. Páez (Museo Herpetológico de la Universidad de Antioquia), F. Castro (Universidad del Valle), O. Castaño (Colección de Reptiles, Instituto de Ciencias Naturales, Universidad Nacional de Colombia), Hno. L. A. Zamudio (Museo del Colegio San José, Medellín), G. Rivas (Museo de Historia Natural La Salle), G. Colli (Colecao Herpetológica da Universidade de Brasilia).

Los colegas V. H. Serrano, G. de Pérez, E. Stashenko, P. Wooding, y estudiantes coautores de los trabajos mencionados: R. Caicedo, J. C. Galeano, A. Jerez, D. Gómez, F. Carreño, S. Vieira, F. Leal, E. Rueda. Los estudiantes que colaboraron en la colecta y mantenimiento de las lagartijas, J. C. Galeano, R. Caicedo, J. Ortega, D. Zárate, F. Leal, O. Tarazona, Ezequiel González, Juan Carlos Afanador, P. Sánchez, Nelsy Pinto, J. P. Ramírez, R. M. Torres. J. Anaya. La Corporación Autónoma de Santander y el Ministerio del Ambiente por los permisos de recolección y acceso a material genético (No. 8, 31 Marzo, 2009, No 0753, 20th Abril, 2010).

\section{Referencias}

Blackburn, D.G. 1992. Convergent evolution of viviparity, matrotrophy, and specializations for fetal nutrition in reptiles and other vertebrates. American Zoology 32: 313-321.

Biazik, J. M., M. B. Thompson, C. R. Murphy. 2009 Lysosomal and alkaline phosphatase activity indicate macromolecule transport across the uterine epithelium in two viviparous skinks with complex placenta. Journal of Experimental Zoology. Part B, Molecular and Developmental Evolution $312817-826$.

Blackburn, D. G., L. J. Vitt. 1992. Reproduction in viviparous South American lizard of the genus Mabuya. In W. C. Hamlett (ed.), Reproductive Biology of South American Vertebrates, pp. 150-164. Springer-Verlag, New York.

Blackburn, D. G., I. P. Callard. 1997. Morphogenesis of placental membranes in the viviparous, placentotrophic lizard Chalcides chalcides (Squamata: Scincidae). Journal of Morphology 232:35-55.

Brandley, M. C., R. L. Young, D. L. Warren, M. B. Thompson, G. P. Wagner. 2012. Uterine gene expression in the livebearing lizard, Chalcides ocellatus, reveals convergence of squamate reptile and mammalian pregnancy mechanisms. Genome Biology and Evolution 4 394-411.

Caicedo-Portilla, R., V.H. Serrano-Cardozo, M. P. RamírezPinilla. 2010. Diet, microhabitat use and daily activity patterns of an Andean population of Mabuya (Squamata: Scincidae). South American Journal of Herpetology 5 (1): 57-63.
Callard, I. P., S. M. Ho. 1987. Vitellogenesis and viviparity. In: I. Chester Jones, P. M. Ingleton, and J. G. Phillips (Eds.), Fundamentals of Comparative Vertebrate Endocrinology. Plenum Press, 8: 257-282.

Dyke, G., G. W. Kaiser, 2010. Cracking a developmental constraint: egg size and bird evolution. En: Proceedings of the VII International Meeting of the Society of Avian Paleontology and Evolution, ed. W.E. Boles and T.H. Worthy. Records of the Australian Museum 62 (1): 207-216.

Flemming, A. F., W. R. Branch. 2001. Extraordinary case of matrophy in the African skink Eumecia anchietae. Journal of Morphology 247: 264-287.

Gómez, D., M. P. Ramírez-Pinilla. 2004. Ovarian histology of the viviparous matrotrophic lizard Mabuya mabouya (Squamata, Scincidae). Journal of Morphology 259: 90-105.

Grant, T. 2007. The Platypus. 4th Edition. CSIRO Publishing: Collingwood, Vic.

Griffith, O. W, B. Ujvari, K. Belov, M. B. Thompson. 2013. Placental lipoprotein lipase (LPL) gene expression in a placentotrophic lizard, Pseudemoia entrecasteauxii. Journal of Experimental Zoology. Part B, Molecular and Developmental Evolution 320: 465-470.

Guerrero, S.M., M. L. Calderón, G. R. De Pérez, M. P. RamírezPinilla. 2003. Annual reproductive activity of Caiman crocodilus fuscus in captivity. Zoo Biology 22: 121-133.

Hernádez-Franyutti, A., M. C. Uribe-Aranzábal, L. J. Guillette Jr. 2005. Oogenesis in the viviparous matrotrophic lizard Mabuya brachypoda. Journal of Morphology 265: 152-164.

Jerez, A. 1998. Placentación en poblaciones de Mabuya cf mabouya. Trabajo de grado, Escuela de Biología, Universidad Industrial de Santander. 120 pp.

Jerez, A., M. P. Ramírez-Pinilla. 2001. The allantoplacenta of Mabuya mabouya (Sauria, Scincidae). Journal of Morphology 249: 132-146.

Jerez, A., M. P. Ramírez-Pinilla. 2003. Morphogenesis of extraembryonic membranes and placentation of Mabuya mabouya. Journal of Morphology 258: 158-178.

Leal, F., M. P. Ramírez-Pinilla. 2008. Morphological variation in the allantoplacenta within the genus Mabuya (Squamata: Scincidae). Anatomical Record. 291: 1124-1139.

Leal, F., M. P. Ramírez-Pinilla. 2010. Evolution and development of the extraembryonic membranes in lizards: Heterochronies and Placentotrophy. Herpetological Conservation and Biology 5 (2): 297-310.

Murphy, K., S. Hudson, G. Shea. 2006. Reproductive seasonality of three cold-temperate viviparous skinks from southeastern Australia. Journal of Herpetology 40 (4): 454-464.

Ramírez-Pinilla, M.P. 2006. Placental transfer of nutrients during gestation in an Andean population of the highly matrotrophic lizard genus Mabuya (Squamata: Scincidae). Herpetological Monographs 20: 194-204. 
Ramírez-Pinilla, M. P. 2010. Matrotrofía en reptiles escamados. Pp. 109-136. En: Hernández-Gallegos, O., Méndez de la Cruz, F.R. \& Méndez-Sánchez, J.F. (compiladores): Reproducción en reptiles: morfología, ecología y evolución. Editorial Katrina, S.A., Departamento editorial de la Universidad Autónoma del Estado de México. Toluca, México. ISBN: 978-607-422-088-9.

Ramírez-Pinilla, M. P., V. H. Serrano, J. C. Galeano. 2002. Annual reproductive activity of Mabuya mabouya (Squamata, Scincidae). Journal of Herpetology 36: 667-677.

Ramírez-Pinilla, M. P., G. De Pérez, J. F. Carreño-Escobar. 2006. Allantoplacental ultrastructure of an Andean population of Mabuya (Squamata, Scincidae). Journal of Morphology 267: $1227-1247$.

Rocha, C. F. D., D. Vrcibradic. 1999. Reproductive traits of two sympatric viviparous skinks (Mabuya macrorhyncha and Mabuya agilis) in a Brazilian Restinga habitat. Herpetological Journal 9: 43-53.

Selwood, L. 2007. Marsupial oocytes, fertilization and embryonic development can provide useful tools to study developmental mechanisms. IUBMB Life 59 (10): 617 - 621.

Serrano-Cardozo, V. H., M. P. Ramírez-Pinilla, J. E. Ortega, L. A. Cortes. 2007. Annual reproductive activity of Gonatodes albogularis (Squamata: Gekkonidae). South American Journal of Herpetology 2 (1): 31-38.

Stewart, J., Thompson, M. 2003. Evolutionary Transformations of the Fetal Membranes of Viviparous Reptiles: A Case Study of Two Lineages. Journal of Experimental Zoology. Part A: Ecological Genetics and Physiology 299A (1): 13-32.
Thompson, M., J. Stewart, B. Speake, M. Hosie, C. Murphy. 2002. Evolution of viviparity: what can Australian lizards tell us? Comparative Biochemistry and Physiology. Part B: Biochemistry and Molecular Biology 131 (4): 631-643.

Van Dyke, J. W., M. C. Brandley, M. B. Thompson. 2014. The evolution of viviparity: molecular and genomic data from squamate reptiles advance understanding of live birth in amniotes. Reproduction 147: R15-R26.

Vieira, S H., G. Romero De Pérez, M. P. Ramírez-Pinilla. 2007. Invasive cells in the placentome of Andean populations of Mabuya: an endotheliochorial contribution to the placenta? Anatomical Record 290: 1508-1518.

Vieira, S H., G. Romero De Pérez, M. P. Ramírez-Pinilla. 2010. Ultrastructure of the ovarian follicles in the highly placentotrophic Andean lizard Mabuya sp (Squamata: Scincidae). Journal of Morphology: 271 (6): 738-749.

Vitt, L. J., D. G. Blackburn. 1983. Reproduction in the lizard M. heathi (Sauria: Scincidae): a commentary on viviparity in new world Mabuya. Canadian Journal of Zoology 61: 2798-2806.

Vitt, L. J., D. G. Blackburn. 1991. Ecology and life history of the viviparous lizard Mabuya bistriata (Scincidae) in the Brazilian Amazon. Copeia 1991:916-927.

Vrcibradic, D., C. F. D. Rocha. 1998. Reproductive cycle and life-history traits of the viviparous skink Mabuya frenata in Southeastern Brazil. Copeia 1998:612-619.

Wooding, P., M. P. Ramírez-Pinilla, A.S. Forhead. 2010. Functional studies of the placenta of the lizard Mabuya sp. (Scincidae) using immunocytochemistry. Placenta 31 (8): 675-685. 